Independent Journal of Management \& Production (IJM\&P)

v. 2, n. 1, January - June 2011

ISSN: 2236-269X

DOI: $10.14807 /$ ijmp.v2i1.25

\title{
UTILIZANDO O MÉTODO TODIM PARA AVALIAR AS MELHORES EMPRESAS PARA TRABALHAR
}

\author{
Renato Teixeira da Silva \\ Faculdade de Engenharia de Guaratinguetá (UNESP/FEG )- Brazil \\ E-mail: renatovr@gmail.com
}

Nilson Brandalise Escola de Engenharia Industrial Metalúrgica de Volta Redonda (UFF) - Brazil E-mail: nilson_01@yahoo.com.br

Christian Augusto Guimarães Vargas Carneiro Escola de Engenharia Industrial Metalúrgica de Volta Redonda (UFF) - Brazil E-mail: christian@metal.eeimvr.uff.br

Submission: 12/01/2011

Accept: 17/03/2011

\section{RESUMO:}

Este artigo expõe uma nova proposta de ordenação das 5 melhores empresas para trabalhar em termos de qualidade do ambiente de trabalho e qualidade na gestão de pessoas utilizandose do método TODIM como suporte científico para tal. A ordenação inicial, proposta pela revista Você/SA Exame, utiliza como método classificador o índice de felicidade no trabalho, uma soma ponderada de índices resultantes da percepção dos funcionários, das práticas da empresa e da constatação feita por jornalistas em visitas às empresas. Este trabalho, que utiliza o método TODIM, considera esses itens como critérios de avaliação e realiza uma análise comparativa das alternativas. Ao final, é executada uma comparação entre as ordenações e uma análise de sensibilidade, analisando as especificidades de cada método de ordenação e a consistência de seus resultados.

Palavras-Chaves: Método TODIM; Apoio Multicritério à Decisão; Avaliação de Empresas

\section{INTRODUÇÃO}

Os métodos de Apoio Multicritério à Decisão (AMD) são aplicados em casos onde na presença de múltiplos critérios, busca-se selecionar, ordenar, classificar ou descrever alternativas, (Roy \& Bouyssou, 1993; Romero, 1993; Vincke, 1989), dependendo da problemática abordada. A maioria dos métodos do AMD podem ser classificados dentro de duas grandes Escolas: a Americana, buscando agregar as avaliações em uma grande síntese; $\mathrm{e}$ a Francesa (ou Européia), com a noção de relação de superação. Alguns outros métodos, por exemplo, o TODIM Gomes e Lima (1992; apud Gomes et al. (2004), contém elementos técnicos dessas duas escolas, denominados métodos híbridos.

Este artigo objetiva aplicar o método TODIM para avaliar o desempenho em termos de práticas de gestão e satisfação dos trabalhadores de um conjunto de empresas do Brasil. O artigo está organizado da seguinte forma: na seção 2 aborda-se a teoria dos prospectos, fundamental para a compreensão do método TODIM, que é explicado na seção 3. Nas seções seguintes apresentam-se o estudo de caso e as conclusões, respectivamente. 


\section{A TEORIA DOS PROSPECTOS}

A Teoria dos Prospectos (Kahneman \& Tversky, 1979) busca modelar o comportamento do ser humano no que tange a tomada de decisão de risco. Através de pesquisas observou-se que o ser humano apresenta uma maior aversão ao risco em situações de ganho, adotando uma postura mais conservadora. Prefere-se então ganhar pouco, mas que este ganho seja certo, ao invés do risco de ganhar mais ou não ter ganho algum. Já em situações com risco de perda, o ser humano apresenta certa inclinação ao risco. Prefere-se o risco de perder mais ou não perder a uma certeza de perda menor. (Maranhão, 2006; Gomes \& Rangel, 2007)

A Função de Valor que representa esta teoria pode ser vista na Figura 1. No primeiro quadrante, onde são representados os ganhos, uma curva côncava descreve a aversão ao risco de ganho. Já no terceiro quadrante, onde são representadas as perdas, uma curva convexa e mais íngreme representa a inclinação ao risco de perda.

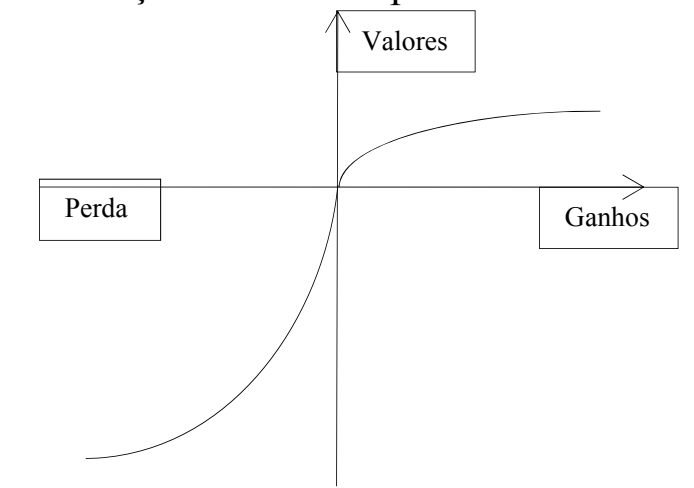

Figura 1 - Função de Valor da Teoria dos Prospectos.

Fonte: Maranhão (2006; Gomes e Rangel (2007)

\section{O MÉTODO TODIM}

O método multicritério TODIM (TOmada de Decisão Interativa Multicritério) Gomes e Lima (1992; apud Gomes et al. (2004) objetiva ordenar um grupo de alternativas avaliando-as segundo um conjunto de critérios de decisão, descrevendo assim um método para resolver problemas do tipo $\mathrm{P} \gamma$. O diferencial do método TODIM é a utilização de uma medida global calculável através da aplicação da Função de Valor da Teoria dos Prospectos. Praticamente todos os outros métodos multicritério têm por objetivo do seu tomador de decisão buscar o valor máximo de alguma media global de valor.

Considera-se um conjunto de $\mathrm{n}$ alternativas a serem ordenadas na presença de $\mathrm{m}$ critérios quantitativos ou qualitativos e assume-se que um desses critérios será considerado o critério de referência. Deve-se então, com o auxílio de especialistas, para cada um dos critérios qualitativos $\mathrm{Cj}$, avaliar de cada uma das $\mathrm{n}$ alternativas. Para a avaliação das alternativas em relação aos critérios quantitativos, utilizamos o valor da performance da alternativa no critério, por exemplo, o valor em reais da receita bruta de uma empresa, o consumo de energia de uma máquina em $\mathrm{kW}$.

O método TODIM necessita que valores da avaliação das alternativas em relação a cada critério sejam numéricos e normalizados. Sendo assim, a avaliação feita através dos critérios qualitativos deve ser transformada para uma escala ordinal, obtendo assim a matriz de avaliação das alternativas. Ambas as avaliações em critérios quantitativos e qualitativos são normalizadas utilizando-se, por exemplo, da divisão da avaliação da alternativa i pela soma das avaliações das alternativas para cada critério, formando a matriz dos valores das 
DOI: $10.14807 /$ ijmp.v2i1.25

alternativas normalizadas $\mathrm{P}=[\mathrm{Pnm}]$, onde $\mathrm{n}$ representa $\mathrm{o}$ número de alternativas e $\mathrm{m} \mathrm{o}$ número de critérios, mostrado na Tabela 1.

Tabela 1 - Matriz dos Valores das Alternativas Normalizadas

\begin{tabular}{ccccccc}
\hline \multirow{2}{*}{ Alternativas } & \multicolumn{7}{c}{ Critérios } \\
\cline { 2 - 7 } & $\boldsymbol{C}_{1}$ & $\boldsymbol{C}_{2}$ & $\ldots$ & $\boldsymbol{C}_{j}$ & $\ldots$ & $\boldsymbol{C}_{\boldsymbol{m}}$ \\
\hline $\boldsymbol{A}_{\boldsymbol{1}}$ & $P_{11}$ & $P_{12}$ & $\ldots$ & $P_{1 j}$ & $\ldots$ & $P_{1 m}$ \\
$\boldsymbol{A}_{\boldsymbol{2}}$ & $P_{21}$ & $P_{22}$ & $\ldots$ & $P_{2 i}$ & $\ldots$ & $P_{2 m}$ \\
$\ldots$ & $\ldots$ & $\ldots$ & $\ldots$ & $\ldots$ & $\ldots$ & $\ldots$ \\
$\boldsymbol{A}_{\boldsymbol{i}}$ & $P_{i 1}$ & $P_{i 2}$ & $\ldots$ & $P_{i j}$ & $\ldots$ & $P_{i m}$ \\
$\ldots$ & $\ldots$ & $\ldots$ & $\ldots$ & $\ldots$ & $\ldots$ & $\ldots$ \\
$\boldsymbol{A}_{\boldsymbol{n}}$ & $P_{n 1}$ & $P_{n 2}$ & $\ldots$ & $P_{n j}$ & $\ldots$ & $P_{n m}$ \\
\hline
\end{tabular}

Após atribuir pesos para cada um dos critérios e normalizá-los, deve-se calcular a matriz de dominância parcial e a matriz de dominância final. Os decisores devem indicar um critério r como critério referência para os cálculos de acordo com a importância relativa de cada critério. O peso de cada critério é determinado pelos decisores em uma escala numérica e normalizado. Então, a taxa de substituição wrc é calculada a partir da divisão do peso do critério c pelo peso do critério referência $r$. A utilização da taxa de substituição permite trazer para uma mesma escala todas as medidas de comparação por pares de performance. A medida da dominância de cada alternativa Ai, incorporada a teoria dos Prospectos, é dada pela Eq. (1):

$$
\delta\left(A_{i}, A_{j}\right)=\sum_{c=1}^{m} \Phi_{c}\left(A_{i}, A_{j}\right) \forall(i, j)
$$

onde:

$$
\Phi_{c}\left(A_{i}, A_{j}\right)=\mid \begin{array}{lc}
\sqrt{\frac{w_{r c}\left(P_{i c}-P_{j c}\right)}{\sum_{c=1}^{m} w_{r c}}} & \text { se }\left(P_{i c}-P_{j c}\right)>0, \\
0 & \text { se }\left(P_{i c}-P_{j c}\right)=0, \\
\frac{-1}{\theta} \sqrt{\frac{\left(\sum_{c=1}^{m} w_{r c}\right)\left(P_{j c}-P_{i c}\right)}{w_{r c}}} & \text { se }\left(P_{i c}-P_{j c}\right)<0 .
\end{array}
$$

sendo:

- $\delta\left(A_{i}, A_{j}\right)$ a medida de dominância relativa da alternativa i sobre a alternativa j;

- m o número de critérios;

- c um critério qualquer, $c=1, \ldots, m$;

- wrc a taxa de substituição do critério c pelo critério de referência r;

- Pic e Pjc, respectivamente, os valores normalizados das alternativas i e j em relação ao critério c;

- $\theta$ o fator de atenuação de perdas.

O fator $\Phi_{c}\left(A_{i}, A_{j}\right)$ representa a contribuição do critério c à função $\delta\left(A_{i}, A_{j}\right)$, quando se compara as alternativas i e j. O valor da diferença entre Pic e Pic representará diferentes situações para a função $\delta\left(A_{i}, A_{j}\right)$ e para cada uma delas, uma diferente equação deverá ser utilizada para se obter o fator $\Phi_{c}\left(\mathrm{~A}_{\mathrm{i}}, \mathrm{A}_{\mathrm{j}}\right)$ : em situações de ganho para a função, onde a diferença é positiva, utiliza-se a Eq. (2); em situações de equivalência, onde a diferença é 
nula, utiliza-se a Eq. (3); em situações de perda, onde a diferença é negativa, utiliza-se a Eq. (4).

Obtida a matriz de dominância final $\delta\left(A_{i}, A_{j}\right)$ através da soma das $m$ matrizes de dominância parcial $\Phi_{c}\left(A_{i}, A_{j}\right)$, ela então é normalizada, utilizando-se da Eq. (5), para obter o valor global de cada alternativa. A ordenação das alternativas provém da ordenação de seus respectivos valores globais.

$$
\xi_{i}=\frac{\sum_{j=1}^{n} \delta\left(A_{i}, A_{j}\right)-\min \sum_{j=1}^{n} \delta\left(A_{i}, A_{j}\right)}{\max \sum_{j=1}^{n} \delta\left(A_{i}, A_{j}\right)-\min \sum_{j=1}^{n} \delta\left(A_{i}, A_{j}\right)}
$$

Por conseguinte, o método TODIM determina uma escolha, pelo fato de ordenar todas as alternativas, procedentes das preferências expressas por um decisor ou grupo de decisores. Uma análise de sensibilidade deve ser considerada avaliando não só as implicações caso seja alterado o fator de atenuação de perdas, mas também, dependendo da finalidade, os pesos dos critérios, o critério de referência ou ainda a avaliação das alternativas.

\section{ESTUDO DE CASO}

O grupo Abril publica anualmente relação das melhores empresas para trabalhar do Brasil, denominado "GUIA VOCÊ/SA EXAME AS MELHORES EMPRESAS PARA VOCÊ TRABALHAR". Sua principal fonte de informações são as respostas a questionários enviados a funcionários das empresas participantes, e um conjunto Book de Evidências. A coleta destas informações é feita sem muitas dificuldades pois para participar da pesquisa é necessário que as empresas se inscrevam para participar da pesquisa, demonstrando interesse no seu resultado.

O critério classificador considerado foi o Índice de Felicidade no Trabalho (IFT), que é composto por três índices que representam:

- A percepção dos funcionários;

- As práticas de gestão da empresa;

- A validação das informações por jornalistas em visita feita a empresa.

Esta pesquisa busca ordenar, segundo o método TODIM, as 5 melhores empresas da relação VOCÊ/SA EXAME publicada em 2009.

\subsection{Definição dos critérios}

- Índice de Qualidade do Ambiente de Trabalho: obtido através da resposta dos funcionários a questionários que visavam constatar:

o Identificação com a empresa

o Satisfação e Motivação;

o Visão de desenvolvimento;

o Aprovação das lideranças. 
- Índice de Qualidade na Gestão de Pessoas: obtido através da resposta dos funcionários a questionários e um conjunto de evidências que visavam constatar os níveis de:

o Estratégia e gestão;

o Cidadania empresarial;

o Políticas e práticas de:

- Carreira profissional;

- Desenvolvimento;

- Remuneração e Benefícios;

- Saúde.

- O índice gerado pela validação dos jornalistas foi desconsiderado por não ser fornecido pela revista na pesquisa.

\subsection{Peso dos Critérios}

O peso dos critérios será o mesmo utilizado no cálculo do IFT e podem ser vistos na Tabela 2.

Tabela 2 - Peso dos Critérios

\begin{tabular}{|c|c|c|c|}
\hline Critério & Descrição & Peso & $\begin{array}{c}\text { Peso } \\
\text { Normalizado }\end{array}$ \\
\hline $\mathrm{C}_{1}$ & Índice de Qualidade do Ambiente de Trabalho & 0,70 & 0,737 \\
\hline $\mathrm{C}_{2}$ & Índice de Qualidade na Gestão de Pessoas & 0,25 & 0,263 \\
\hline
\end{tabular}

\subsection{As Alternativas do Processo de Decisão}

Neste estudo foram avaliadas as 5 empresas brasileiras que apresentaram os melhores desempenhos na ordenação do guia VOCÊ/SA EXAME, que são as alternativas do processo decisório. A Figura 2 associa as alternativas com as 5 melhores empresas do guia.

\begin{tabular}{|c|l|}
\hline A1 & CATERPILLAR \\
\hline A2 & MASA DA AMAZÔNIA \\
\hline A3 & VOLVO DO BRASIL \\
\hline A4 & LABORATÓRIO SABIN \\
\hline A5 & EUROFARMA \\
\hline
\end{tabular}

Figura 2 - Alternativas do Processo de Decisão

A Tabela 3, contendo a Avaliação das Alternativas segundo cada Critério, apresenta uma avaliação completa das alternativas estudadas na analise em relação aos critérios selecionados. Ela também contém o valor normalizado dessa avaliação, que é fundamental para a implementação do método TODIM.

Tabela 3 - Avaliação das Alternativas segundo cada Critério 
DOI: $10.14807 /$ ijmp.v2i1.25

\begin{tabular}{|c||c|c||c|c|}
\hline \multirow{2}{*}{ Alternativas } & \multicolumn{2}{|c||}{ Critérios } & \multicolumn{2}{c|}{ Critérios Normalizados } \\
\cline { 2 - 5 } & C1 & C2 & C1 & C2 \\
\hline A1 & 92,1 & 89,9 & 0,206 & 0,200 \\
\hline A2 & 92 & 89,7 & 0,206 & 0,200 \\
\hline A3 & 90,7 & 93,6 & 0,203 & 0,208 \\
\hline A4 & 87,2 & 87,9 & 0,195 & 0,196 \\
\hline A5 & 85,1 & 88,5 & 0,190 & 0,197 \\
\hline
\end{tabular}

Nesse estudo de caso, o valor definido para o fator de atenuação de perdas $\theta$ foi de 1 . A aplicação do método TODIM resulta em um valor global normalizado para cada alternativa, apresentado na Tabela 4. A ordenação das alternativas, obtida através do valor global normalizado de cada alternativa também está presente na nesta tabela.

Tabela 4 - Valores Globais Normalizados e Ordenação das Alternativas

\begin{tabular}{|c|c|c|c|}
\hline Alternativas & $\square \mathbf{i}$ & $\square \mathbf{i}$ & Ordenação \\
\hline A1 & 0,155 & 0,917 & 2 \\
\hline A2 & 0,061 & 0,835 & 3 \\
\hline A3 & 0,251 & 1,000 & 1 \\
\hline A4 & $-0,831$ & 0,059 & 4 \\
\hline A5 & $-0,899$ & 0,000 & 5 \\
\hline
\end{tabular}

\subsection{Análise de Sensibilidade}

Um dos métodos de analisar a sensibilidade do método TODIM é a partir da variação do valor fator de atenuação de perdas (Gomes e Rangel, 2007). Neste trabalho, a variação deste valor não gera alterações na ordenação obtida, como pode ser visto na Tabela 5. Isso demonstra que a ordenação é, segundo o método, consistente.

Tabela 5 - Valores Globais Normalizados e Ordenação das Alternativas

\begin{tabular}{|c|c|c|c|c|c|c|c|c|c|}
\hline \multirow{2}{*}{ Ordem } & \multicolumn{3}{|c|}{$=1$} & \multicolumn{3}{|c|}{$=5$} & \multicolumn{3}{|c|}{$\square=10$} \\
\hline & Alternativas & $\square \mathbf{i}$ & $\square \mathbf{i}$ & Alternativas & $\square \mathbf{i}$ & $\square \mathbf{i}$ & Alternativas & $\square \mathbf{i}$ & $\square \mathbf{i}$ \\
\hline 1 & A3 & 0,251 & 1,000 & A3 & 0,353 & 1,000 & A3 & 0,366 & 1,000 \\
\hline 2 & $\bar{A} 1$ & 0,155 & 0,917 & $\bar{A} 1$ & 0,296 & 0,891 & A1 & 0,314 & 0,882 \\
\hline 3 & $\mathrm{~A} 2$ & 0,061 & 0,835 & $\mathrm{~A} 2$ & 0,253 & 0,806 & A2 & 0,277 & 0,797 \\
\hline 4 & A4 & $-0,831$ & 0,059 & A4 & $-0,119$ & 0,088 & A4 & $-0,030$ & 0,098 \\
\hline 5 & A5 & \begin{tabular}{|l|}
$-0,899$ \\
\end{tabular} & 0,000 & A5 & $-0,165$ & 0,000 & A5 & $-0,073$ & 0,000 \\
\hline
\end{tabular}

\section{CONCLUSÃo}

Esta pesquisa realizou a comparação de duas ordenações das cinco melhores empresas para trabalhar do país. Uma proposta pelos editores do guia VOCÊ/SA EXAME e, uma 
outra determinada através do emprego do método TODIM de Apoio Multicritério à Decisão.

Os critérios empregados nesta avaliação foram: o índice de Qualidade do Ambiente de Trabalho e; o índice de Qualidade na Gestão de Pessoas. Na avaliação realizada pelo guia VOCÊ/SA EXAME empregou-se a soma ponderada dos pesos dos critérios pelos desempenhos das alternativas com respeito a cada critério. Na avaliação realizada através do método TODIM, além de considerar as importâncias dos critérios realiza-se uma comparação par a par das alternativas para posteriormente agregar essas avaliações num critério de síntese.

Verifica-se através dos resultados que a única diferença na ordenação foi a alteração da Alternativa A3 para a primeira posição. Essa diferença de ordenação é justificada tecnicamente devido ao método empregado pelo guia VOCÊ/SA EXAME ser compensatório, já o método TODIM não é. Um outro fator foi a falta o índice de avaliação gerado a partir da visita dos jornalistas às empresas. Apesar de possuir um peso muito menor que os outros critérios $(0,05)$, a partir dele seria possível justificar a ordenação gerada pelo guia VOCÊ/SA EXAME.

Com esta pesquisa foi possível comparar o emprego de um método de ordenação utilizado pelo guia VOCÊ/SA EXAME, com o método TODIM de Apoio Multicritério à Decisão. A continuação desta pesquisa pode ser realizada através do emprego do método de Kendall para verificar a tendência entre as duas ordenações, ou ainda a utilização de outros métodos, da escola americana ou francesa, para verificação de suas particularidades.

\section{REFERÊNCIAS}

GOMES, L.F.A.M., LIMA, M.M.P.P. TODIM: Basics and application to multicriteria ranking of projects with environmental impacts. Foundations of Computing and Decision Sciences 16 (4), 113-127, $1992 \mathrm{a}$.

GOMES, L.F.A.M., LIMA, M.M.P.P. From modeling individual preferences to multicriteria ranking of discrete alternatives: A look at Prospect Theory and the additive difference model. Foundations of Computing and Decision Sciences 17 (3), 171-184, 1992b.

GOMES, L.F.A.M., RANGEL, L.A.D. An application of the TODIM method to the multicriteria rental evaluation of residential properties, European Journal of Operational Research, doi:10.1016/j.ejor.2007.10.046, 2007.

GUIA VOCÊ/SA EXAME As melhores empresas para você trabalhar. São Paulo: Abril, 2009. Disponível em: < http://vocesa.abril.com.br/melhoresempresas/> . Acesso em: 15 mai 2010.

KAHNEMAN, D., TVERSKY, A. Prospect theory: An analysis of decision under risk. Econometrica 47, 263-292, 1979.

MARANHÃO, F. J. C. A exploração de gás natural em Mexilhão: análise multicritério pelo método TODIM. 2006. 92 f. Dissertação (Mestrado Profissionalizante em Administração) - Faculdade de Economia e Finanças, IBMEC, Rio de Janeiro, 2006.

ROMERO, C. Teoría de la decisión multicriterio: Conceptos, técnicas y aplicaciones. Madrid: Alianza Editorial S.A., Alianza Universidad Textos, p. 195, 1993.

ROY, B., BOUYSSOU, D. , Aide Multicritère à la Décision: Méthodes et Cas. Paris: Ed. Econômica, p. 695, 1993.

TROTTA, L.T.F., NOBRE, F.F., GOMES, L.F.A.M. Multi-criteria decision making - An approach to setting priorities in health care. Statistics in Medicine 18, 3345-3354, 1999.

VINCKE, P. L'aide multicritère à la dècision. Bruxelles: Éditions de 1'Université de Bruxelles, p. 179, 1989. 